\title{
INVESTIGACIONES
}

\section{Los blogs en la formación docente inicial}

\author{
Blogs in initial teacher training
}

Blogs na formação inicial de professores

\author{
Carme Pinya ${ }^{1}$, Gemma Tur ${ }^{2}$, Maria Rosa Rosselló \\ ${ }^{1}$ Universidad de las Islas Baleares, +34971173144, carme.pinya@uib.es \\ ${ }^{2}$ Universidad de las Islas Baleares, +34971398025, gemma.tur@uib.es \\ ${ }^{3}$ Universidad de las Islas Baleares, +439713289, mrosa.rossello@uib.es
}

\section{RESUMEN}

En este artículo se analiza y valora cómo el alumnado percibe el uso del blog como herramienta de aprendizaje. El estudio se basa en una muestra de 70 alumnos y un cuestionario autovalorativo como instrumento de investigación. Entre los resultados obtenidos en el estudio, destaca que el blog es percibido como una herramienta que ayuda a la reflexión, que facilita el proceso de enseñanza-aprendizaje y que potencia el desarrollo de la competencia digital. Cuanto más reflexivo sea el blog y más ayuda propicie en el proceso de enseñanza-aprendizaje, aumentan las posibilidades de uso en el futuro profesional del alumnado. Contrariamente, se apunta a que aunque el blog ayuda al desarrollo de la competencia digital, ello no garantiza su uso futuro. A partir de estos resultados, se propone compaginar el blog con estrategias de escritura reflexiva, con un feedback de carácter formativo y con el uso de redes sociales.

Palabras clave: blog, Educación Superior, formación docente inicial, práctica reflexiva

\section{ABSTRACT}

In this article the perceptions of future teachers' on blogs as a learning tool are analyzed and assessed. The study is based on a sample of 70 students and on a questionnaire of self-assessment as the research instrument. Data obtained highlight that a blog is perceived as a tool that enhances reflection, facilitates the teaching-learning process, and empowers the development of digital skills. The more reflexive the blog and the more helpful it is for the learning process, the more possibilities there are for its use in students' future careers. However, it is also argued that although the blog is perceived positively as a tool that can empower the development of digital skills, this is not a guarantee for its future use. From data obtained, some recommendations are given such as scaffolding the blog with reflexive writing strategies, formative feedback and the use of the social media.

Key words: blog, Higher Education, initial teacher training, reflective practice.

\section{RESUMO}

Analisa-se e avalia-se como os alunos percebem o uso do blog como ferramenta de aprendizagem. O estudo é baseado em uma mostra de 70 alunos e teve um questionário autoavaliativo como instrumento de pesquisa. Entre os resultados, destaca-se que o blog é percebido como uma ferramenta que ajuda na reflexão, que facilita o processo ensino-aprendizagem e que melhora o desenvolvimento da competência digital. Quanto mais reflexivo for o blog, mais ajudará no processo ensino-aprendizagem, aumentando as possibilidades de seu uso futuro na vida profissional dos estudantes. Destaca-se que, ainda que o blog ajude no desenvolvimento da competência digital, isso não garante o seu uso futuro. A partir de estes resultados, propõe-se combinar o blog com estratégias de escrita reflexiva, com feedbacks de caráter formativo e com o uso das redes sociais.

Palavras-chave: blog, Ensino Superior, formação inicial de professores, prática reflexiva. 


\section{INTRODUCCIÓN}

Tras más de dos décadas, el profesorado muestra todavía un bajo dominio en alfabetización digital y tecnológica (Almerich, Suárez-Rodríguez, Belloch y Bo, 2011). Diversos estudios (Teo, 2008; Teo, Lee y Chai, 2008; Teo, 2009; Boza, Tirado, Ramón y Guzmán-Franco, 2010; Contreras y Arancibia, 2013) constatan además que la integración de las herramientas tecnológicas depende de las actitudes que manifiesta el docente, estando éstas, a su vez, estrechamente condicionadas por las creencias y las experiencias que vive en su período de formación inicial (Hermans, Tondeur, Braak y Valcke, 2008; Garrido, Contreras y Miranda, 2013).

Desde esta perspectiva, la incorporación de las Tecnologías de la Información y la Comunicación (TIC) a la formación inicial docente resulta como mínimo imprescindible. Sin embargo, este proceso no puede realizarse de cualquier manera. En numerosas investigaciones se ha observado que el blog es una herramienta potente cuando está al servicio de la mejora de la reflexión (Parkes y Kajder, 2010; Deng y Yuen, 2010; Prestridge, 2014; Dos y Demir, 2013; Isakovic y McNaught, 2013; Yang, 2009) y de los procesos de enseñanza-aprendizaje (Cabero, López y Ballesteros, 2009; Deng y Yuen, 2010; Halic, Lee, Paulus y Spence, 2010). Con todo, la reflexión no es un proceso fácil para el alumnado, que requiere de ayuda y andamiaje para evolucionar desde niveles más descriptivos a otros más críticos y profundos (Prestridge, 2014; Jenson, 2011; Portillo, Iranzo y Rosselló, 2013)

Aunque la denominación de TIC incluye un amplio espectro de herramientas, este artículo se centra en el uso del blog en el contexto universitario. El blog permite ampliar las clásicas limitaciones de espacio y tiempo de la educación tradicional (Cabero et al. 2009; Aguaded, López Meneses y Alonso, 2010). Se trata de un recurso "híbrido" donde se mezclan comentarios, enlaces de tipo profesional, reflexiones y anécdotas, con lugares y otras herramientas de la red (Santoveña, 2011, 60). A nivel técnico, es un instrumento de fácil uso (Fonseca, 2009), sencillo y económico (González Sánchez y García Muiña, 2011). Además, es accesible desde cualquier lugar, eficiente para la organización de contenido, permite la interactividad, la creación de enlaces permanentes y la sindicación.

En opinión de Cabero et al. (2009: 3), los edublogs pueden ser usados al amparo de un amplio abanico de estrategias metodológicas: "discusiones, resolución de problemas o análisis de casos, entrevistas a expertos, elaboración de proyectos grupales o confección de portafolios electrónicos". Por su parte, Bartholomew, Jones y Glassman (2012) remarcan que permiten a los alumnos investigar el universo del conocimiento para aportar a la propia comunidad bloguera. Para Santoveña (2011), los blogs son, fundamentalmente, herramientas de colaboración y participación, ejemplificando el cambio de paradigma docente que está experimentando la universidad.

El análisis bibliográfico muestra como puntos fuertes del blog los rasgos siguientes:

a) Colaboración y creación de comunidad. Los blogs potencian el sentido de comunidad y la colaboración entre los miembros del grupo (Halic, Lee, Paulus, y Spence, 2010; Top, 2012; Aguaded, López Meneses y Alonso, 2010), promoviendo la comunicación horizontal, la motivación para la participación, y la expresión individual y colectiva (Santoveña, 2011), ayudando a desarrollar habilidades sociales y cívicas (Redecker et al., 2009).

b) Reflexión. Como portafolios electrónicos, facilitan la documentación y la reflexión sobre la evolución que sigue el aprendizaje (Chuang, 2010; Deng y Yuen, 2010; Conole y Alevizou, 
2010; Barrett, 2011; Cambridge, 2010; Tur y Urbina, 2012) y las competencias profesionales (Deng y Yuen, 2010; Bartolomé, Canó y Companyó, 2011; Rosselló y Pinya, 2013).

c) Escritura. Mejoran los procesos de expresión escrita (Arslan y Sahin-Kizil, 2010; Baturay y Daloglu, 2010) y fomentan una actitud positiva hacia el proceso de escribir (Marsden y PiggotIrvine, 2012).

d) Autoría. Afianzan el sentido de responsabilidad y propiedad (Redecker et al. 2009), pudiéndose convertir en la sede de la identidad digital del aprendiz (Castañeda y Adell, 2001).

e) Autonomía y evaluación. Favorecen la evaluación continua (González Sánchez y García Muiña, 2011; Conole y Alevizou, 2010) y potencian la colaboración para la evaluación entre pares (Dippold, 2009).

f) Uso de múltiples lenguajes e incremento de la interacción social. Permiten integrar audio e imagen (Deng y Yuen, 2010); amplían las posibilidades para la reflexión, con la creación de vídeos (Parkes y Kadjer, 2010; Parkes, Dredger y Hicks, 2013); posibilitan hacer comentarios y enlazar a otros sitios webs, lo que facilita el aprendizaje colaborativo y cooperativo (Top, 2012).

En cambio, entre los aspectos negativos los estudios hacen referencia básicamente a tres puntos: una gran mortalidad en su uso (González Sánchez y García Muiña, 2011); problemas relacionados con el respeto a la autoría en el contenido que se publica (Tekinarslan, 2008); y el enorme volumen de trabajo que supone, tanto para el alumnado como para el profesorado (Martínez Gimeno y Hermosilla, 2010; Rosselló y Pinya, 2013).

En los últimos años, se observa una incipiente línea de investigación sobre la integración de las TIC en la práctica docente. El desarrollo de las competencias digitales del futuro profesorado es clave para promover una visión de cambio en el uso de las TIC y la Web 2.0 en educación (Bower, Highfield, Furney, Mowbray, 2013; Kidd, 2013).

A su vez, la literatura recoge diversas investigaciones relacionadas con las percepciones del alumnado cuando utiliza blogs en su aprendizaje. Avci y Askar (2012), en un estudio que compara el uso de blogs y wikis, concluyen que ambas herramientas son percibidas positivamente, aunque la wiki es considerada como una herramienta más útil y que genera menor ansiedad entre el alumnado. Goktas y Demirel (2012) apuntan que los alumnos valoran positivamente los blogs y los consideran una herramienta potente para la mejora de su competencia digital. En la misma dirección, otras investigaciones (Halic et al., 2010; Top, 2012) hallan resultados similares y coinciden en que el alumnado valora positivamente la experiencia de aprendizaje con blogs, ya que facilita la colaboración en la construcción de conocimiento, ayudando a la compresión de los contenidos trabajados. Finalmente, Deng y Yuen $(2010,2012)$ observan que los estudiantes de educación encuentran en el blog una plataforma adecuada para la autoexpresión, la reflexión y la interacción con otros.

En esta última línea de investigación situamos el presente artículo, en el que se pretende dar respuesta a una doble interrogante: ¿Cómo percibe el alumnado el uso del blog cuando lo utiliza en el contexto académico? y ¿Qué percepciones determinan que el alumnado use el blog en su futuro profesional?

En respuesta a estos interrogantes, se plantean las siguientes hipótesis:

1. El alumnado percibe el blog como una herramienta que facilita la reflexión.

2. El alumnado percibe el blog como un instrumento de apoyo en el proceso de 
enseñanza-aprendizaje.

3. El alumnado percibe el blog como una ayuda en la adquisición y desarrollo de la competencia digital.

4. El alumnado percibe el blog como una herramienta que será utilizada en su futuro profesional.

5. Las dimensiones de análisis se relacionan positivamente con la dimensión Reflexión.

6. El alumnado afirma que continuará con el blog en su futuro profesional cuando lo percibe como herramienta de reflexión.

7. El alumnado afirma que continuará con el blog en su futuro profesional cuando lo percibe como instrumento de apoyo en el proceso de aprendizaje.

8. El alumnado afirma que continuará con el blog en su futuro profesional cuando lo percibe como herramienta que facilita el desarrollo de su competencia digital.

\section{DISEÑO DE LA INVESTIGACIÓN}

\subsection{CONTEXTO}

La investigación se lleva a cabo durante el curso académico 2012-2013, en el marco de una asignatura de tercer curso del estudio de grado de Educación Infantil de la Universitat de les Illes Balears. Esta asignatura es de segundo semestre y se imparte bajo una modalidad semipresencial, al mismo tiempo que los alumnos están cursando el Practicum en las escuelas infantiles.

\subsection{PARTICIPANTES}

En el curso 2012-2013 se matriculan a la asignatura un total de 102 alumnos, de los cuales 70 participan en el estudio, lo que representa un 68,6\% del total. El proceso de muestreo es intencional.

En cuanto a las características generales de la muestra, podemos destacar que:

- Se respetan los criterios de densidad de población de cada isla: 14,3\% de Menorca, 27,1\% de Ibiza y 58,6\% de Mallorca.

- La muestra está compuesta por un alumnado procedente de dos vías de acceso: un 48,65\% de alumnos acceden mediante selectividad y un 38,6\% acceden mediante formación profesional. El resto $(12,9 \%)$ no indicaron su vía de acceso en el cuestionario.

- La mayoría de los sujetos encuestados son mujeres $(91,4 \%)$, aunque esto responde a la distribución normal de la población en los estudios de Grado de Educación Infantil.

- Un 55,7\% de los encuestados tiene entre 20 y 24 años; un $28,6 \%$ tiene entre 25 y 30 años, y sólo un $15,7 \%$ tiene más de 31 años. La mediana de edad es de 25,9 años y la desviación típica 6,765 .

\subsection{INSTRUMENTO}

Se opta por el cuestionario como instrumento de recogida de datos. Se trata de un cuestionario auto-informe diseñado expresamente y que recoge una síntesis de las 
valoraciones que aparecen en el marco teórico y la adaptación de diversos ítems de los cuestionarios de Lin (2008) y Teo (2008). Consta de 20 preguntas, agrupadas en cuatro dimensiones: Reflexión, Proceso de aprendizaje, Competencia digital y Uso del blog en el futuro profesional. Los ítems del cuestionario se plantean con una escala de valoración Likert, con puntuaciones comprendidas entre 1 y 5 , en la que 1 es nada y 5 mucho.

El cuestionario es sometido a un proceso de contrastación, a partir de un Panel Delphi formado por 8 profesores universitarios conocedores de la materia; se obtiene un $100 \%$ de respuesta y un $90 \%$ de concordancia. En segundo lugar, se lleva a cabo una prueba piloto con un grupo de 20 alumnos, se procede a la reformulación de dos ítems y se obtiene una coincidencia total entre la visión del encuestador y la de los alumnos participantes.

La fiabilidad de consistencia interna del instrumento se estimó mediante el coeficiente Alfa de Cronbach, que fue de 0.88 .

\subsection{PROCEDIMIENTO}

La explicación y aplicación del cuestionario para la obtención de datos se realiza enmarcada dentro de las sesiones de trabajo habitual de los estudios de grado de Maestro en Educación Infantil. Los datos son recogidos por el profesorado de las asignaturas, mediante el pase de los cuestionarios al alumnado. En la sesión de aplicación del cuestionario el profesorado explica el proceso de cumplimentación, así como los objetivos de la investigación. Se recalca el carácter anónimo de las respuestas y se resuelven las dudas antes de comenzar la tarea de cumplimentar el cuestionario.

Una vez recogidos los datos, éstos son procesados. Para el tratamiento y análisis de la información, se utiliza el software de tratamiento de datos estadístico SPSS (Statistical Package for the Social Sciences) en su última versión. Se realiza un análisis estadístico descriptivo, un análisis de correlaciones bivariadas y, finalmente, la prueba $\mathrm{T}$ para muestras independientes.

\section{RESULTADOS}

En la primera fase de análisis de resultados, y con la finalidad contrastar las cuatro primeras hipótesis, se lleva a cabo una explotación estadística descriptiva de las frecuencias de los diferentes ítems, y se obtienen datos relativos a la media y la desviación típica de cada una de ellos.

Tabla 1. Estadísticas descriptivas

\begin{tabular}{|c|c|c|}
\hline & Media & Desviación típica \\
\hline Reflexión & 3,70 & 0,593 \\
\hline Proceso aprendizaje & 3,23 & 0,522 \\
\hline Competencia digital & 3,09 & 0,776 \\
\hline Uso del blog en el futuro profesional & 3,65 & 0,935 \\
\hline
\end{tabular}


Las puntuaciones demuestran que el alumnado percibe el blog como herramienta que facilita la reflexión, sirve de apoyo a los procesos de enseñanza-aprendizaje, ayuda a la adquisición y desarrollo de la competencia digital, y que será utilizada en su futuro profesional, confirmándose de este modo las cuatro primeras hipótesis. Las puntuaciones más elevadas corresponden a la dimensión de Reflexión y a la del Uso del blog en el futuro profesional, en este orden.

En la segunda fase del análisis, los resultados muestran que la dimensión Reflexión correlaciona de manera positiva con las tres dimensiones restantes. En cambio, no hay correlación entre las dimensiones Proceso de aprendizaje, Competencia Digital y Uso del blog en el futuro profesional.

Tabla 2. Correlaciones entre las diferentes dimensiones.

\begin{tabular}{|l|c|c|c|c|c|}
\hline \multicolumn{2}{|c|}{} & Reflexión & $\begin{array}{c}\text { Proceso } \\
\text { aprendizaje }\end{array}$ & $\begin{array}{c}\text { Competencia } \\
\text { Digital }\end{array}$ & $\begin{array}{c}\text { Uso del blog en el } \\
\text { futuro profesional }\end{array}$ \\
\hline \multirow{2}{*}{ Reflexión } & r de Pearson & 1 & & & \\
\cline { 2 - 6 } & Sig. & & & & \\
\hline \multirow{2}{*}{$\begin{array}{l}\text { Proceso } \\
\text { pprendizaje }\end{array}$} & r de Pearson & 0,568 & 1 & & \\
\cline { 2 - 7 } & Sig. & 0,000 & & & \\
\hline \multirow{2}{*}{$\begin{array}{l}\text { Competencia } \\
\text { digital }\end{array}$} & r de Pearson & 0,516 & 0,310 & 1 & \\
\cline { 2 - 7 } & Sig. & 0,001 & 0,079 & & \\
\hline $\begin{array}{l}\text { Uso del blog } \\
\text { en el futuro } \\
\text { profesional }\end{array}$ & r de Pearson & 0,304 & 0,212 & 0,188 & \\
\cline { 2 - 7 } & Sig. & 0,020 & 0,132 & 0,228 & \\
\hline
\end{tabular}

En la tercera fase del análisis, y con el objetivo de verificar las últimas hipótesis, se aplica la prueba $\mathrm{T}$ para muestras independientes, tomando como variable de agrupación la dimensión relativa al Uso del blog en el futuro profesional.

De acuerdo con la hipótesis seis, se constata que existen diferencias estadísticamente significativas entre la dimensión Reflexión y el Uso del blog en el futuro profesional. Se obtiene una media superior cuando el alumnado percibe el blog como herramienta que facilita la reflexión.

Se confirma la hipótesis siete, ya que existen diferencias estadísticamente significativas entre la dimensión Proceso de aprendizaje y Uso del blog en el futuro profesional. Se obtiene una media superior cuando el alumnado percibe el blog como un instrumento de apoyo al proceso de enseñanza-aprendizaje.

En cambio, la hipótesis ocho queda rechazada, ya que no existen diferencias estadísticamente significativas entre el Uso del blog en el futuro profesional y el hecho de percibirlo como herramienta que facilita el desarrollo de su competencia digital. 
Tabla 3. Comparación de medias según el uso del blog en el futuro.

\begin{tabular}{|l|c|c|c|c|c|c|c|}
\hline & $\begin{array}{c}\text { Si será } \\
\text { utilizado }\end{array}$ & $\begin{array}{c}\text { Desviación } \\
\text { típica }\end{array}$ & $\begin{array}{c}\text { No será } \\
\text { utilizado }\end{array}$ & $\begin{array}{c}\text { Desviación } \\
\text { típica }\end{array}$ & $\mathrm{t}$ & $\mathrm{gl}$ & Sig. \\
\hline Reflexión & 3,96 & 0,54 & 3,53 & 0,57 & 2,912 & 51,505 & 0,005 \\
\hline $\begin{array}{l}\text { Proceso } \\
\text { aprendizaje }\end{array}$ & 3,47 & 0,35 & 3,13 & 0,55 & 2,744 & 43,637 & 0,009 \\
\hline $\begin{array}{l}\text { Competencia } \\
\text { digital }\end{array}$ & 3,19 & 0,89 & 3,04 & 0,71 & 0,571 & 26,070 & 0,573 \\
\hline
\end{tabular}

\section{DISCUSIÓN}

El estudio muestra que el alumnado participante percibe el blog positivamente, como una herramienta útil para la reflexión, que ayuda en el proceso de enseñanza-aprendizaje y que sirve para mejorar la competencia digital. Coincide en este sentido con otras investigaciones desarrolladas en el ámbito internacional, tales como Halic et al., (2010) y Top (2012). En la misma dirección que los trabajos de Deng y Yuen (2010, 2012), los participantes encuentran en el blog una plataforma adecuada para la autoexpresión y la reflexión; el estudio arroja resultados similares al correlacionar positivamente la dimensión Reflexión con el proceso de enseñanza-aprendizaje.

Por otra parte, el estudio demuestra también que cuando el alumnado percibe el blog como herramienta de reflexión y como ayuda al proceso de enseñanza-aprendizaje, decide continuar con el blog en su futuro profesional. En este sentido, la investigación va más allá del estudio realizado por Wang, Lin y Liao (2012), donde se relaciona la continuidad del blog con las características personales del alumnado.

Se aporta así una nueva dimensión del uso del blog en el contexto universitario, al vincularlo con procesos de reflexión. Futuras investigaciones deberían profundizar en este aspecto, ubicando al alumnado en escenarios TIC que cuestionan actitudes y creencias, fuertemente influenciadas por experiencias iniciales, tal como indican Hermans, Tondeur, Braak y Valcke (2008), y que promuevan además el uso de las TIC para la innovación didáctica (Garrido, Contreras y Miranda, 2013). Al constatar que la utilización del blog en el futuro profesional depende de dos variables básicas (la reflexión y la percepción de utilidad), se abre el camino para orientar el tipo de tareas y actividades que puede proponer el profesorado universitario cuando persigue potenciar la escritura reflexiva a través del blog. En este sentido, Deng y Yuen (2012) recomiendan usar además algunas de las estrategias siguientes:

- Impulsar los llamados early adopters, para que actúen como dinamizadores del grupo clase.

- Que el profesorado use ejemplos extraídos de los blogs, comente y aporte comentarios en blogs de alumnos, etc.

- Alumnos con habilidades TIC como tutores de sus compañeros

En futuras ediciones conviene contemplar la necesidad de compaginar el blog con el uso del feed-back y con estrategias que potencien la escritura reflexiva, así como la 
recomendación que realizan Zhu y Procter (2012) de compaginar el uso de los blogs con redes sociales, como Twitter y Facebook.

\section{CONCLUSIONES}

Una vez analizados los resultados obtenidos, se extraen las siguientes conclusiones: primera, el alumnado universitario valora el uso del blog positivamente, ya que considera que es una herramienta útil para la reflexión, que ayuda al proceso de enseñanza-aprendizaje y que contribuye a incrementar su competencia digital; segunda, existe una relación entre determinadas percepciones (la reflexión y la utilidad) del alumnado y el uso del blog en su futuro profesional.

Estas conclusiones guardan una estrecha relación con estudios de análogas características. A su vez, sugieren algunas recomendaciones y propuestas relacionadas de intervención para el futuro, como se ha destacado en el apartado de discusión.

Finalmente, cabe reconocer que el estudio presenta una clara limitación relacionada con el instrumento utilizado. Se prevé complementar las limitaciones propias del cuestionario con una nueva recogida de datos de carácter cualitativo utilizando los grupos de discusión focal.

\section{REFERENCIAS BIBLIOGRÁFICAS}

Almerich, G.; Suárez-Rodríguez, J.M.; Belloch, C. y Bo, R.M. (2011). Las necesidades formativas del profesorado en TIC: perfiles formativos y elementos de complejidad. RELIEVE, vol.17, n.2, Recuperado el 13/05/2014 de http://www.uv.es/RELIEVE/v17n2/RELIEVEv17n2_1.htm

Aguaded, J.I., López Meneses, E.; Alonso, L. (2010). Innovating with blogs in university courses: a qualitative study. The New Educational Review, vol. 22, n.3-4, 103-116. Recuperado el 22/04/2014 de http://rabida.uhu.es/dspace/bitstream/handle/10272/6385/Innovating_with_ blogs.pdf?sequence $=2$

Arslan, R.; Sahin-Kizil, A. (2010). How can the use of blog software facilitate the writing process of english language learners? Computer Assisted Language Learning, vol.23, n.3, 183-197.

Avci, U.; Askar, P. (2012). The Comparison of the Opinions of the University Students on the Usage of Blog and Wiki for Their Courses. Educational Technology \& Society, vol.15, n.2, 194-205 Recuperado el 29/02/2014 de http://www.ifets.info/journals/15_2/17.pdf

Bartholomew, M., Jones, T.; Glassman, M. (2012). A community of voices: Educational blog management strategies and tools. TechTrends, vol. 56, n.4, 19-25.

Bartolomé, A.; Cano, E.; Compañó, P. (2011) Los blogs como instrumento para la evaluación de competencias en los entornos de prácticas. En Pagés, T. (Coord.), Buenas prácticas docentes en la universidad. Modelos y experiencias en la Universidad de Barcelona. Barcelona: ICE/ Octaedro

Barrett, H. (2011). Balancing the two faces of eportfolios. En S. Hirtz, K. Kelly (Eds.), Education for a digital world. Innovations in education, vol. 2 (pp. 291-310). British Columbia Ministry of Education. Recuperado el 3/4/2014 de http://www.openschool.bc.ca/info/edu/7540006133_2. pdf

Baturay, M. H.; Daloglu, A. (2010). E-portfolio assessment in an online english language course. Computer Assisted Language Learning, vol. 23, n.5, 413-428.

Bower, M.; Highfield, K.; Furney, P.; Mowbray, L. (2013). Supporting Pre-Service Teachers' 
Technology-Enabled Learning Design Thinking through Whole of Programme Transformation. Educational Media International, vol.50, n.1, 39-50.

Boza, Á.; Tirado, R. ; Guzmán-Franco, M.D. (2010). Creencias del profesorado sobre el significado de la tecnología en la enseñanza: influencia para su inserción en los centros docentes andaluces. RELIEVE, vol.16, n.1. Recuperado el 27/02/2014 de http://www.uv.es/RELIEVE/ v16n1/RELIEVEv16n1_5.htm

Cabero, J., López, E.; Ballesteros, C. (2009). Experiencias universitarias innovadoras con blogs para la mejora de la praxis educativa en el contexto europeo. RUSC. Revista De Universidad $Y$ Sociedad Del Conocimiento, vol.6, n.2. Recuperado el 22/04/2014 de http://www.raco.cat/ index.php/RUSC/article/view/140242/191428

Cambridge, D. (2010). Eportfolios for Lifelong Learning and Assessment. San Francisco: JosseyBass.

Castañeda, L.; Adell, J. (2011). El desarrollo profesional de los docentes en entornos personales de aprendizaje (PLE). En R. Roig Vila y C. Laneve (Eds.), La práctica educativa en la sociedad de la información. Innovación a través de la investigación. La pratica educativa nella società dell'informazione. L'innovazione attraverso la ricerca (pp. 83-95). Alcoi-Brescia: Marfil i La Scuola Editrice.

Chuang, H. H. (2010). Weblog-based electronic portfolios for student teachers in taiwan. Educational Technology Research and Development, vol.58, n.2, 211-227.

Conole, G.; Alevizou, P. (2010). A literature review of the use of Web 2.0 tools in Higher Education. HEA Academy, York, UK. Recuperado el 11/01/2014 de http://oro.open.ac.uk/23154/

Contreras, P.; Arancibia, M. (2013). Aprendizaje y TIC: Innovaciones Didácticas para transformar contextos educativos. Estudios Pedagógicos (Valdivia), vol.39, Número Especial I, pp. 5-6. Recuperado el 27/06/2014 de http://www.scielo.cl/scielo.php?pid=S071807052013000300001\&script=sci_arttext

Deng, L.; Yuen, A. H. K. (2010). Towards a framework for educational affordances of blogs. Computers \& Education, vol.56, n.2.

Deng, L.; Yuen, H. K. (2012). Understanding student perceptions and motivation towards academic blogs: An exploratory study. Australasian Journal of Educational Technology, vol.28, n.1, 4866. Recuperado el 10/07/2014 de www.ascilite.org.au/ajet/ajet28/deng.pdf

Dippold, D. (2009). Peer feedback through blogs: Student and teacher perceptions in an Advanced German class. ReCALL- European Association for Computer Assisted Language Learning, vol.21, n.1, 18- 36 .

Dos, B. \& Demir, S. (2013) The analysis of the blogs created in a blended course through the reflective thinking perspective. Educational Sciences: Theory \& Practice, vol.13, n.2, 1335-1344. Recuperado el 08/03/2014 de http://www.edam.com.tr/kuyeb/en/makale. asp?ID $=850 \&$ act $=$ detay

Fonseca, M. C. (2009). Edublogs: Blog's para educar. En C. Castaño (Coord.), Web 2.0. El uso de la web en la sociedad del conocimiento. Investigación e implicaciones educativas (pp. 85-114). Venezuela : Universidad Metropolitana. Recuperado el 04/06/2014 de http://www. tecnologiaedu.us.es/tecnoedu/images/stories/castanio20.pdf

Garrido, J.; Contreras, D.; Miranda, C. (2013). Análisis de la disposición pedagógica de los futuros profesores para usar las TIC. Estudios Pedagógicos (Valdivia), vol. 39, Número Especial I, pp. 59-74. Recuperado de http://www.scielo.cl/pdf/estped/v39nEspecial/art05.pdf

Goktas, Y.; Demirel, T. (2012). Blog-enhanced ICT courses: Examining their effects on prospective teachers' ICT competencies and perceptions. Computers \& Education, vol.58, n.3, 908-917.

González Sánchez, R.; García Muiña, F.E. (2011). Recursos eficaces para el aprendizaje en entornos virtuales en el Espacio Europeo de Educación Superior: análisis de los edublogs. ESE. Estudios sobre educación, vol.20, 161-180. Recuperado el 22/04/2014 de http://dspace.unav.es/dspace/ bitstream/10171/18416/2/ESE\%20161-180.pdf 
Halic, O., Lee, D., Paulus, T.; Spence, M. (2010). To blog or not to blog: Student perceptions of blog effectiveness for learning in a college-level course. The Internet and Higher Education vol.13, $n .4$.

Hermans, R; Tondeur, J.; van Braak, J.; Valcke, M. (2008). The impact of primary school teachers' educational beliefs on the classroom use of computers. Computers \& Education, vol.51, 14991509.

Isakovic, A.; McNaught, A. (2013) Supporting Learning Through the Use of Self-Reflection Blogs: A study of the experience of blended learning students in the United Arab Emirates. Open Praxis, vol.5, n.4, 355-363. Recuperado el 29/03/2014 de http://openpraxis.org/index.php/OpenPraxis/ article/view/89

Jenson, J. (2011). Promoting self-regulation and critical reflection through writing students use of electronic portfolio. International Journal of EPortfolio, vol.1, n.1, 49-60. Recuperado de www. theijep.com/pdf/IJEP19.pdf

Kidd, W. (2013). Framing Pre-Service Teachers' Professional Learning Using Web2.0 Tools: Positioning Pre-Service Teachers as Agents of Cultural and Technological Change. Professional Development in Education, vol.39, n.2, 260-272.

Lin, Q. (2008). Preservice teachers' learning experience of constructing eportfolios online. The Internet and Higher Education, vol.11, n.3-4, 194-200.

Marsden, N.; Piggot-Irvine, E. (2012). Using blogging and laptop computers to improve writing skills on a vocational training course. Australasian Journal of Educational Technology, vol.28, n.1, 30-47. Recuperado el 25/05/2014 de www.ascilite.org.au/ajet/ajet28/marsden.html

Martínez Gimeno, A.; Hermosilla, J. (2010). El blog como herramienta didáctica en el Espacio Europeo de Educación Superior. Pixel Bit: Revista de medios y educación, vol.38, 165 - 175. Recuperado el 14/05/2014 de a www.sav.us.es/pixelbit/actual/13.pdf

Parkes, K., Kajder, S. (2010). Eliciting and assessing reflective practice: A case study in web 2.0 technologies. International Journal of Teaching and Learning in Higher Education, vol.22, n.2, 218-228. Recuperado el 11/02/2014 de www.isetl.org/ijtlhe/pdf/IJTLHE947.pdf

Parkes, K., Dredger, K.; Hicks, D. (2013). ePortfolio as a Measure of Reflective Practice. International Journal of ePortfolio, vol.3, n.2, 99-115. Recuperado el 27/01/2014 de http://www.theijep.com/ pdf/IJEP110.pdf

Portillo, M.C.; Irazno, P.; Rosselló, M.R. (2013) Autorregulación de aprendizajes en educación superior mediante la escritura de blogs. Revista Iberoamericana de Educación. vol.63, n.2, Recuperado el 22/03/2014 de http://www.rieoei.org/expe/5829Rosello.pdf

Prestridge, S. (2014). Reflective blogging as part of ICT professional development to support pedagogical change. Australian Journal of Teacher Education, vol.39, n.29.

Redecker, C., Ala-Mutka, K., Bacigalupo, M., Ferrari, A., \& Punie, Y. (2009). Learning 2.0: The impact of web 2.0 innovations on education and training in europe. Final Report. European Commission-Joint Research Center-Institute for Porspective Technological Studies, Seville. Recuperado el 05/06/2014 de ftp.jrc.es/EURdoc/JRC55629.pdf

Rosselló, M.R.; Pinya, C. (2013).El blog como herramienta de Reflexión Educativa. II Congreso Internacional de Ciencias de la Educación y del Desarrollo. Santander, 8-11 de octubre de 2013.

Santoveña, S. (2011). Incidencia de los nuevos alfabetismos en la mejora de la calidad de la enseñanza: El caso de los blogs. Aula Abierta, vol.39, n.2, 59-68. Recuperado el 21/03/2014 de http://www. dialnet.unirioja.es/servlet/fichero_articulo?codigo $=3621299 \&$ orden $=0$

Tekinarslan, E. (2008). Blogs: a qualitative investigation into an instructor and undergraduate students' experiences. Australasian Journal of Educational Technology, vol.24, n.4, 402-412. Recuperado el 08/04/2014 de http://www.ascilite.org.au/ajet/ajet24/tekinarslan.pdf

Teo, T. (2008). Pre-service teachers' attitudes towards computer use: A Singapore survey. Australasian Journal of Educational Technology, vol.24, n.4, 413-424. Recuperado el 08/04/2014 de http:// www.ascilite.org.au/ajet/ajet24/teo.pdf 
Teo, T., Lee, C.B.; Chai, C.S. (2008). Understanding pre-service teachers' computer attitudes: applying and extending the technology acceptance model. Journal of Computer Assisted Learning, vol. 24, 128-143.

Teo, T. (2009). Modelling technology acceptance in education: A study of pre-service teachers. Computers \& Education, vol.52, n.2, 302-312.

Top, E. (2012). Blogging as a social medium in undergraduate courses: Sense of community best predictor of perceived learning. The Internet and Higher Education, vol.15, n.1, 24-28.

Tur, G.; Urbina, S. (2012). PLE-based ePortfolios: Towards Empowering Student Teachers' PLEs through ePortfolio Processes. Actas de The PLE Conference 2012, Aveiro, Portugal. Recuperado el 37/01/2014 de http://revistas.ua.pt/index.php/ple/article/view/1438

Wang, Y. S., Lin, H. H.; Liao, Y. W. (2012). Investigating the individual difference antecedents of perceived enjoyment in students' use of blogging. British Journal of Educational Technology, vol. 43, n.1, 139-152.

Yang, S.H. (2009). Using blogs to enhance critical reflection and community of practice. Educational Technology \& Society, vol.12, n.2, 11-21. Recuperado el 20/06/2014 de http://www.ifets.info/ journals/12_2/2.pdf

Zhu, Y.; Procter, R. (2012). Use of blogs, Twitter and Facebook by PhD Students for Scholarly Communication: a UK study. Actas del New Media Communication Association Annual Conference, 6-8 Diciembre 2012. Recuperado el 20/06/2014 de https://www.escholar.manchester. ac.uk/api/datastream?publicationPid=uk-ac-man-scw: $187789 \&$ datastreamId=FULL-TEXT. PDF 
